\title{
Clinical study of nimotuzumab combined with concurrent radiochemotherapy for treatment of locally advanced cervical cancer
}

This article was published in the following Dove Press journal:

Cancer Management and Research

\author{
Wenli Chen' \\ Tao $\mathrm{Li}^{\mathrm{I}}$ \\ Jian Wang' \\ Long Liang' \\ Dandan Huang' \\ Gaoshu Yan' \\ Ye $\operatorname{Tian}^{2}$ \\ Xiaoli Zhang ${ }^{3}$ \\ Wei Zhang ${ }^{4}$ \\ 'Department of Radiation Oncology, \\ Sichuan Cancer Hospital \& Institute, \\ Chengdu, Sichuan 6I004I, People's \\ Republic of China; ${ }^{2}$ Department of \\ Thoracic Tumor Surgery, The Fifth \\ People's Hospital of Chengdu, Chengdu \\ 6III30, People's Republic of China; \\ ${ }^{3}$ Department of Breast and Thyroid \\ Surgery, The Fifth People's Hospital of \\ Chengdu, Chengdu 6III30, People's \\ Republic of China; ${ }^{4}$ Department of \\ Radiation Oncology, Sichuan Cancer \\ Hospital \& Institute, Chengdu 61004I, \\ People's Republic of China
}

Purpose: Nimotuzumab is a humanized monoclonal antibody that targets the epidermal growth factor receptor (EGFR) to inhibit tumor growth. Nimotuzumab has demonstrated desirable therapeutic activity in various types of tumors. However, the benefit of nimotuzumab for the treatment of cervical cancer is not entirely clear. The present study aimed to investigate the effects of nimotuzumab in the presence of CCRT in the first-line treatment of locally advanced cervical cancer (LACC).

Methods: The therapeutic efficacy and side effects of nimotuzumab combined with concurrent radiochemotherapy (CCRT) were retrospectively assessed in inoperable patients with LACC (stage IIb-IIIb) who were treated using CCRT with or without nimotuzumab.

Results: The complete response rate of study group was significantly better than control group ( $78.3 \%$ vs $50 \%, P=0.035)$. The difference in median progression-free survival (PFS) in the two groups was statistically significan (not reach vs 27 months, $P=0.037$ ). Multivariate comparisons of prognostic factors in the two groups indicated that both the Fédération Internationale de Gynécologie et d'Obstétrique (FIGO) stage and combined nimotuzumab treatment affected PFS $(P<0.05)$. Although generally tolerable, grade 3-4 toxicities including leukopenia $(P=0.025)$ and hemoglobin $(P=0.026)$ reduction were more frequent in the control group than those in the study group.

Conclusion: These data suggest that combining nimotuzumab with CCRT for the treatment of LACC resulted in extended PFS and higher complete remission rates, without an increased incidence of adverse events.

Keywords: EGFR monoclonal antibody, Nimotuzumab, cervical cancer, concurrent radiochemotherapy, PFS

\section{Introduction}

Cervical cancer is the fourth most commonly diagnosed malignancy in women and the fourth leading cause of cancer-related death in the world. ${ }^{1}$ Although screening for cervical cancer has improved and most patients undergo surgical therapy in the early stages, patients without timely screening may develop locally advanced cervical cancer (LACC) in which the tumor size is increased and the survival rate is quite low. Additionally, it is difficult to increase the radical radiotherapy dose in advanced cases due to dose limits related to the tolerability of healthy pelvic tissue. The results of five large-scale clinical studies (GOG85, RTOG900, GOG120, GOG123, and SWOG8797) demonstrated that CCRT improved patient treatment outcomes and reduced recurrence and death rates by $30-50 \% .^{2-6}$ Although platinum-based CCRT has become the
Department of Radiation Oncology,

Sichuan Cancer Hospital \& Institute, No.

55, Section 4, South People's Road,

Chengdu, Sichuan 61004I, People's

Republic of China

Tel +862885420630

Fax +862885420630

Email shanheshui200I@I63.com 
standard treatment for LACC, most patients are only partially relieved or relieved for only a short time after therapy ${ }^{7}$ and over $35 \%$ of patients continue to have tumors and experience recurrence and metastasis. This has led researchers and medical staff to focus on improving therapeutic efficacy. As a result, targeted therapy has gained increasing attention as a highly effective approach. The epidermal growth factor receptor (EGFR) is overexpressed in cervical cancer cells. After binding to its ligand, EGFR forms a dimer and activates downstream signal transduction pathways, thereby curbing cell apoptosis and promoting cell formation and angiogenesis. Blocking the signal transduction of EGFR can increase the sensitivity of the tumor to radiotherapy because EGFR is associated with tumor resistance to cytotoxic drugs and radiotherapy. This makes EGFR a desirable target gene for the treatment of cervical cancer. ${ }^{8}$

Nimotuzumab is a monoclonal antibody with a humanization ratio of over $90 \%$. Its benefits include a long circulation half-life, low rate of adverse events, high selectivity, and high humanization ratio. Nimotuzumab competitively inhibits binding of endogenous ligands to EGFR and blocks downstream signal transduction pathways and cytological effects mediated by EGFR, thus interfering with tumor cell proliferation and promoting tumor cell apoptosis. ${ }^{9}$ Nimotuzumab has demonstrated effective outcomes in the treatment of head and neck cell squamous cell carcinoma, ${ }^{10}$ non-small cell lung cancer, ${ }^{11}$ pancreatic cancer, ${ }^{12}$ and many other tumors. These reports show that nimotuzumab is able to stabilize disease for long time periods while causing limited adverse reactions. However, its effects as an EGFR antibody in combination with CCRT in the treatment of advanced cervical cancer have not been investigated in clinical studies.

The present study investigated the effects of nimotuzumab in the presence of CCRT in the treatment of LACC. The retrospective design compared short-term overall response rates, five-year overall survival rates (OS), progression-free survival (PFS), and acute side events between the study and control groups. The results of this study provide additional clinical data and relevant knowledge.

\section{Patient recruitment and inclusion/ exclusion criteria}

Data were collected from patients with LACC treated at Sichuan Cancer Hospital, Chengdu, China between January 2012 and December 2014.
Enrollment criteria: 1) LACC with confirmed clinical stage of IIb -IIIb according to the Fédération Internationale de Gynécologie et d'Obstétrique (FIGO) staging system; 2) at least one lesion before treatment, according to the Response Evaluation Criteria in Solid Tumors (RECIST); 3) able to be treated using CCRT with or without nimotuzumab for at least two cycles; 4) meeting basic requirements for radiotherapy, including a Karnofsky Performance Status score (KPS) $\geq 60$ and an estimated survival of over three months; and 5) patient informed consent obtained together with voluntary acceptance of nimotuzumab treatment.

Exclusion criteria: 1) allergies to the biological agents, 2) absence of measurable lesions, 3) severe organ dysfunction or not suitable for chemotherapy, 4) previous treatment with other anti-tumor strategies, and 5) pregnant or breastfeeding.

All patients were informed about the study procedures and were willing to participate. In accordance with international, national, and local requirements, all patients provided written consent prior to treatment. The study was approved by the Sichuan Cancer Hospital Ethics Committee for Clinical Investigation and carried out in accordance with the ethical principles for medical research of the Declaration of Helsinki.

\section{Treatments}

Study group: CCRT combined with nimotuzumab.

Control group: CCRT only.

Chemotherapy: All patients were administered $135 \mathrm{mg} / \mathrm{m}^{2}$ paclitaxel (Taiji Group, Sichuan Province, China, batch number: 11110040 ) and 50-60 mg/m $\mathrm{m}^{2}$ cisplatin (Jiangsu Hansoh Pharmaceutical Group, Jiangsu province, China, batch number: 140803) during radiotherapy. Each cycle lasted 21-28 days

Nimotuzumab treatment: The first course of nimotuzumab administration was started simultaneously with radiotherapy. Patients were treated once weekly with $200 \mathrm{mg}$ of nimotuzumab (Biotech Pharma Co, Ltd, Beijing, China, batch code: 0120140101) via an intravenous drip infusion. The treatment lasted for 8-12 weeks during which adverse events were strictly monitored and patients were provided appropriate medical care as needed.

Radiotherapy: Patients were placed in the Trendelenburg position, fixed with thermoplastic plastic film, and marked on both the body and film surfaces. Afterward, the patients underwent an enhanced scan with a computed tomography (CT) simulator in the presence of 
a location device. The scanning layer thickness was $3 \mathrm{~mm}$ and the scanning area covered from the T10 inferior edge to $3 \mathrm{~cm}$ below the inferior edge of the sciatic tubercle. Scanning CT images were transmitted to a planning system through a local network. The target areas and organs at risk (OAR) were delineated in $\mathrm{CT}$ images at a workstation.

External beam radiotherapy: The gross tumor volume (GTV) included pelvic lesions visible in magnetic resonance imaging (MRI) and positron emission tomography (PET)-CTs. The clinical target volume (CTV) included the uterine body, para-uterine tissues, vagina and iliac, external iliac, obturator, sacral lymph nodes, and surrounding tissues. The pelvic organs at risk included the small intestine, rectum, bladder, bilateral femoral head, etc. The small intestine was delineated to $1 \mathrm{~cm}$ above the planning target volume (PTV) using a fat window (window level -30, window width 300 ) delineation to better visualize the peri-intestinal tissues. A three-dimensional (3D) margin with $0.5 \mathrm{~mm}$ thickness was added to both GTV and CTV to form the GTV and CTV corresponding planning target volumes (PTV-G and PTV-C, respectively). The PTV-C contained the PTV-G. The OAR included the spinal cord, kidney distension, small intestine, colon, rectum, bladder, pelvic bone, and femoral head. Planning was performed using Masterplan and reverse planning was done with intensity-modulated radiation therapy (IMRT). The dose restriction levels were the PTV-G, PTV-C, small intestine, bladder, and rectum. The prescription doses of PTV-C and PTV-G were 46 Gy for both and each was performed 23 times.

3D internal and external fusion IMRT intracavitary brachytherapy was conducted after external beam radiotherapy using an Ir192 high-dose-rate brachytherapy machine (Nucletron, Holland). The therapy procedure included 10 steps, as follows: 1 . The lesion location was determined based on MRI/CT and gynecological examinations. 2. A negative-pressure body membrane was made and fixed. 3. Three-hundred (300) $\mathrm{mL}$ of physiological saline was injected through a catheter to fill the patient's bladder to reduce radiation exposure to the bladder and small intestine. 4. General disinfection of the perineum and vagina was conducted and an aseptic hole-towel was spread around the vagina. 5. A radiation source applicator was inserted into the vagina with gauze. 6 . A CT-enhanced scan for location was performed and $3 \mathrm{D}$ images of the vagina were delivered to a Nucletron intensity-modulated planning system. 7. The target area, GTV, CTV, and OAR were defined and delineated. In radiotherapy planning, a 95\% iso-dose curve covering $100 \%$ of the PTV was considered acceptable. 8 . The prescription dose was $600 \mathrm{GY} / \mathrm{F}$ for the GTV and $500 \mathrm{GY} / \mathrm{F} * 4 \mathrm{~F}$ for the CTV. 9. An Ir-192 high-dose-rate brachytherapy machine was used. 10. The radiation source applicator and catheter were removed when the treatment was finished.

\section{Evaluation of treatment outcomes and adverse events}

According to World Health Organization (WHO) solid tumor efficacy criteria, a CT scan, general situation assessment, and gynecological examination were performed three months after treatment. The results of the treatment outcome evaluation were divided into four levels: complete response (CR), partial response (PR), stable disease (SD), and progressive disease (PD). Of these, CR and PR were considered objective responses. The complications of radiotherapy were assessed using the Radiation Therapy Oncology Group (RTOG) radiation injury classification criteria.

PFS was defined as the growing time of previous lesions or new lesions from the beginning of the intervention to the time when the lesions could be measured by solid tumor efficacy criteria in CT or MRI determination of disease progression. OS was defined as the time from patient enrollment to the date of death, including death caused by any factor. Adverse events due to medications were recorded prior to every treatment and evaluated by hematology, renal and liver laboratory measures, and physical and psychological examination. Acute adverse events were evaluated using CTCAE 3.0. The follow-up period began four weeks after the last treatment and was conducted once every three months. All patients were followed-up for five years.

\section{Statistical analysis}

Data were analyzed using SPSS software (17.0). Enumeration data were displayed as rates (\%) and statistical differences between groups were evaluated by $x^{2}$ or Fisher's exact tests. Differences between ordinal categorical variables were evaluated by rank-sum tests. Measurement data were described by means \pm standard deviation $(\overline{\mathrm{x}} \pm \mathrm{SD})$ and the differences between groups were evaluated by one-sample $t$-tests. Survival curves were generated using the Kaplan-Meier method and the independent variables were screened by Cox regression 
model analysis. $P$-values $<0.05$ were considered statistically significant.

\section{Results}

In accordance with the study inclusion and exclusion criteria, 53 patients were enrolled in this study. The general patient information is presented in Table 1 . The study group included 23 patients aged $20-65$ years, with a median age of 52 years. The study group included 17 cases of squamous cell carcinoma, three cases of adenocarcinoma and three cases of adenosquamous carcinoma. Seven cases were categorized as stage IIB, six cases as stage IIIa, and 10 cases as stage IIIb. The 30 patients in the control group received only CCRT. This group included 13 cases of squamous cell carcinoma, 13 cases of adenocarcinoma, and four cases of adenosquamous carcinoma. Nine cases were categorized as stage IIb, four as stage IIIa, and 17 as stage IIIb. The ages ranged from 31 to 70 years, with a median age of 51 years. There were no statistically significant differences in age or other general information categories between the treatment and control groups $(P>0.05)$.

\section{Short-term treatment outcomes}

The overall response rates in the study and control groups were $87 \%$ and $73.3 \%$ respectively $\left(\chi^{2}=1.469, P=0384\right)$, a difference that was not statistically significant. The complete response rates were $78.3 \%$ and $50 \%$ respectively, a difference that was statistically significant $\left(\chi^{2}=4.425\right.$, $P=0.035$ ) (Table 2 ). The median time to complete chemo-

Table I Comparison of general conditions of the patients in the study and control groups

\begin{tabular}{|l|l|l|l|}
\hline $\begin{array}{l}\text { Clinical } \\
\text { characteristics }\end{array}$ & $\begin{array}{l}\text { Study group } \\
(\mathbf{n}=\mathbf{2 3})\end{array}$ & $\begin{array}{l}\text { Control group } \\
(\mathbf{n}=\mathbf{3 0 )}\end{array}$ & $\mathbf{P}$ \\
\hline $\begin{array}{l}\text { Age (years, median } \\
\text { value) }\end{array}$ & 52 & $5 \mathrm{I}$ & 0.82 \\
$\begin{array}{l}\text { Pathological type } \\
\text { Squamous cell } \\
\text { carcinoma }\end{array}$ & 17 & 13 & 0.79 \\
$\begin{array}{l}\text { Adenocarcinoma } \\
\text { Adenosquamous } \\
\text { carcinoma }\end{array}$ & 3 & 3 & \\
$\begin{array}{l}\text { Stage } \\
\text { Illb }\end{array}$ & 3 & 4 & \\
Illa & 7 & 6.46 \\
Illb & 6 & 9 & \\
\hline
\end{tabular}

radiotherapy was $3.7 \pm 0.761$ months in the control group and $3.229 \pm 0.737$ months in the study group.

\section{Overall survival rates}

The median follow-up durations in the study and control groups were 46 months (16-60 months) and 42 months (2-60 months), respectively (95\% confidence interval [CI], 29.78-49.22). All patients in both groups were followedup. The three-year OS rates in the study and control groups were $77 \%$ and $60 \%\left(\chi^{2}=1.993, P=0.158\right)$, respectively, and the five-year OS rates were $63.6 \%$ and $36.1 \%$ $\left(\chi^{2}=3.483, P=0.062\right)$, respectively. Neither of these differences was statistically significant. There were seven deaths in the study group and 17 in the control group (Figure 1).

\section{Progression-free survival}

The median PFS of the study group had not been reached during the study period (9-55 months). The median PFS of the control group was 27 months (1-60 months) (95\% CI, 19.19-34.81). The difference in PFS between the two groups was statistically significant $\left(\chi^{2}=4.337, P=0.037\right)$ (Figure 2).

\section{Acute adverse events}

The common acute adverse events in both the study and control groups were leukocyte reduction, thrombocytopenia, and hemoglobin reduction. A small number of patients had confirmed symptoms of impaired liver or kidney function. Patients in both groups were able to tolerate these adverse reactions and no treatment-related deaths occurred. There were statistically significant differences between the two groups in leukocyte reduction and hemoglobin reduction of 3 and 4 grade $(P<0.05)$, but no significant statistical differences were observed for the other side effects (Table 3). Only the highest-grade adverse event was counted. There were no grade 4 toxicities related to liver and kidney function. Grade 3 and 4 toxicities were more frequent in the control group compared to that in the study group. The high incidence of acute adverse events may be due to the low KPS score $(\geq 60)$ in the included patients.

\section{Multivariate analysis}

Multivariate prognostic factor analysis using a Cox proportional hazard model indicated that FIGO stage was an independent indicator of OS. Both FIGO stage and the combination of CRT and nimotuzumab were independent indicators of PFS $(P<0.05)$ (Tables 4 and 5). 
Table 2 Comparisons of short-term response rates in the study and control groups [n (\%)]

\begin{tabular}{|l|l|l|l|l|l|}
\hline Short-term efficacy evaluation & CR & PR & SD & PD & Total \\
\hline Study group & $18(78.3 \%)$ & $2(8.7 \%)$ & $1(4.3 \%)$ & $2(8.7 \%)$ & 23 \\
Control group & $15(50 \%)$ & $7(23.3 \%)$ & $3(10 \%)$ & $5(16.7 \%)$ & 30 \\
Total & 33 & 9 & 4 & 7 & 53 \\
\hline
\end{tabular}

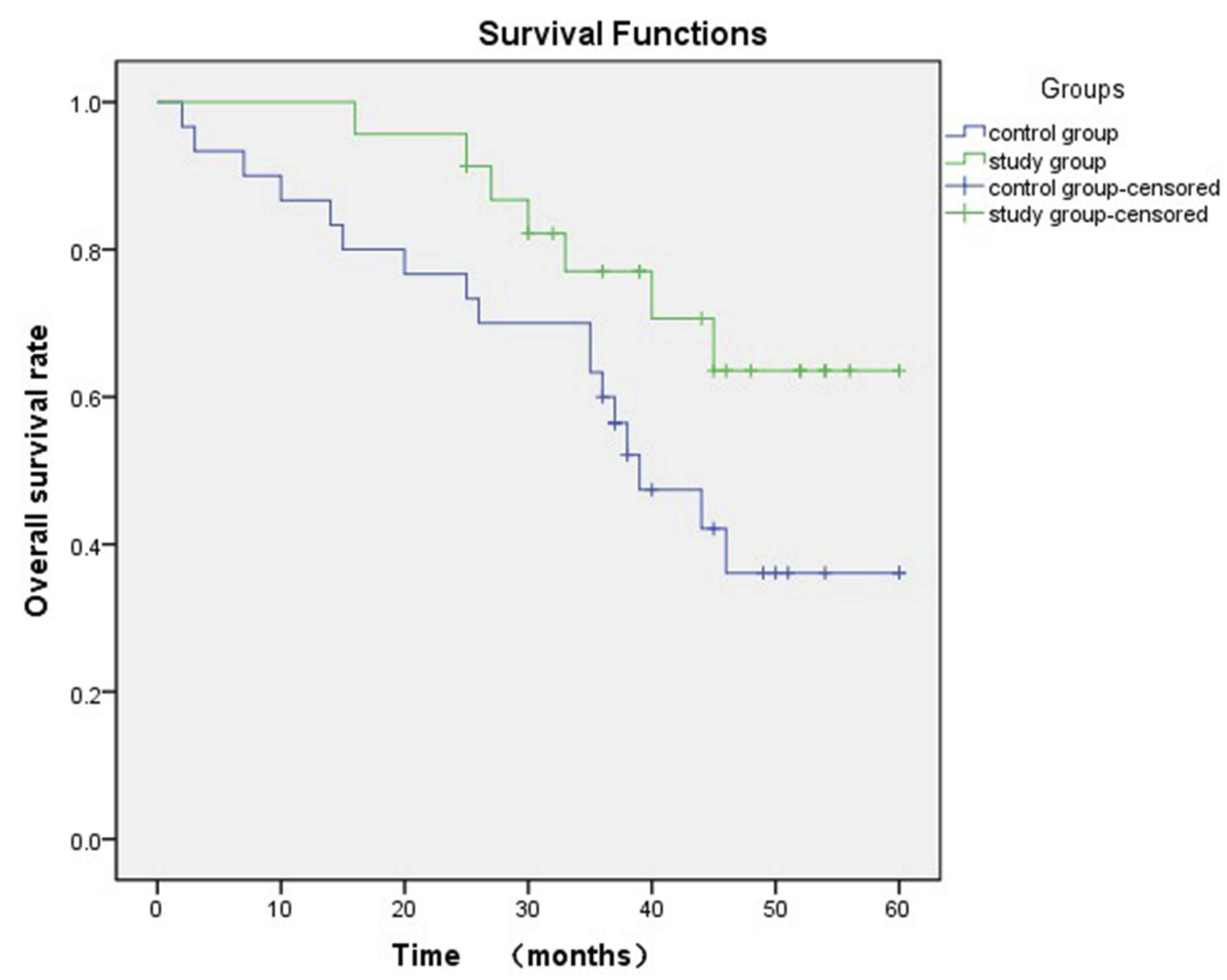

Figure I Kaplan-Meier plots of overall survival rates in the study and control groups.

\section{Discussion}

EGFR, a member of the ErbB (HER) family, is a type of transmembrane glycoprotein. It forms a homodimer or heterodimer with its ligands to trigger cellular signal transduction pathways and then regulates cell differentiation, proliferation, invasion, migration, and angiogenesis. EGFR is overexpressed in a wide range of tumors, including rectal, head and neck, ${ }^{13}$ breast, and cervical cancers. ${ }^{14}$ It is a vital target gene in targeted therapy. A meta-analysis of the correlation between epidermal growth factor overexpression and the prognosis of cervical cancer found that EGFR overexpression was related to OS and disease-free survival (DFS) in cervical cancer and that overexpression of EGFR is likely a predictor of poor prognosis in cervical cancer. ${ }^{15}$ As a consequence, targeted therapy has become a new approach for the treatment of cervical cancer. The currently used drugs including gefitinib, erlotinib, cetuximab, and nimotuzumab all have limited efficacies in improving overall response and recurrence rates in the treatment of advanced cervical cancer, regardless of their use as the only drug or combined with chemotherapy in second- or third-line treatment. ${ }^{8,16-18}$

Based on previous studies of the mechanism of EGFR, the combination of EGFR-targeted drug therapy, radiotherapy, and chemotherapy is currently being explored as a new front-line treatment model for LACC. One phase II clinical study that used erlotinib combined with CCRT to treat stage IIb to IIIb cervical cancer showed that $94.4 \%$ of patients were completely relieved. The three-year OS rate was $80.6 \%$ and the PFS rate was $73.8 \%$, indicating that this treatment is safe and effective. ${ }^{19}$ However, the results of another phase II randomized clinical study that compared cetuximab treatment for stage Ib2-IIIb cervical cancer with and without CCRT showed that cetuximab failed to extend DFS by two 


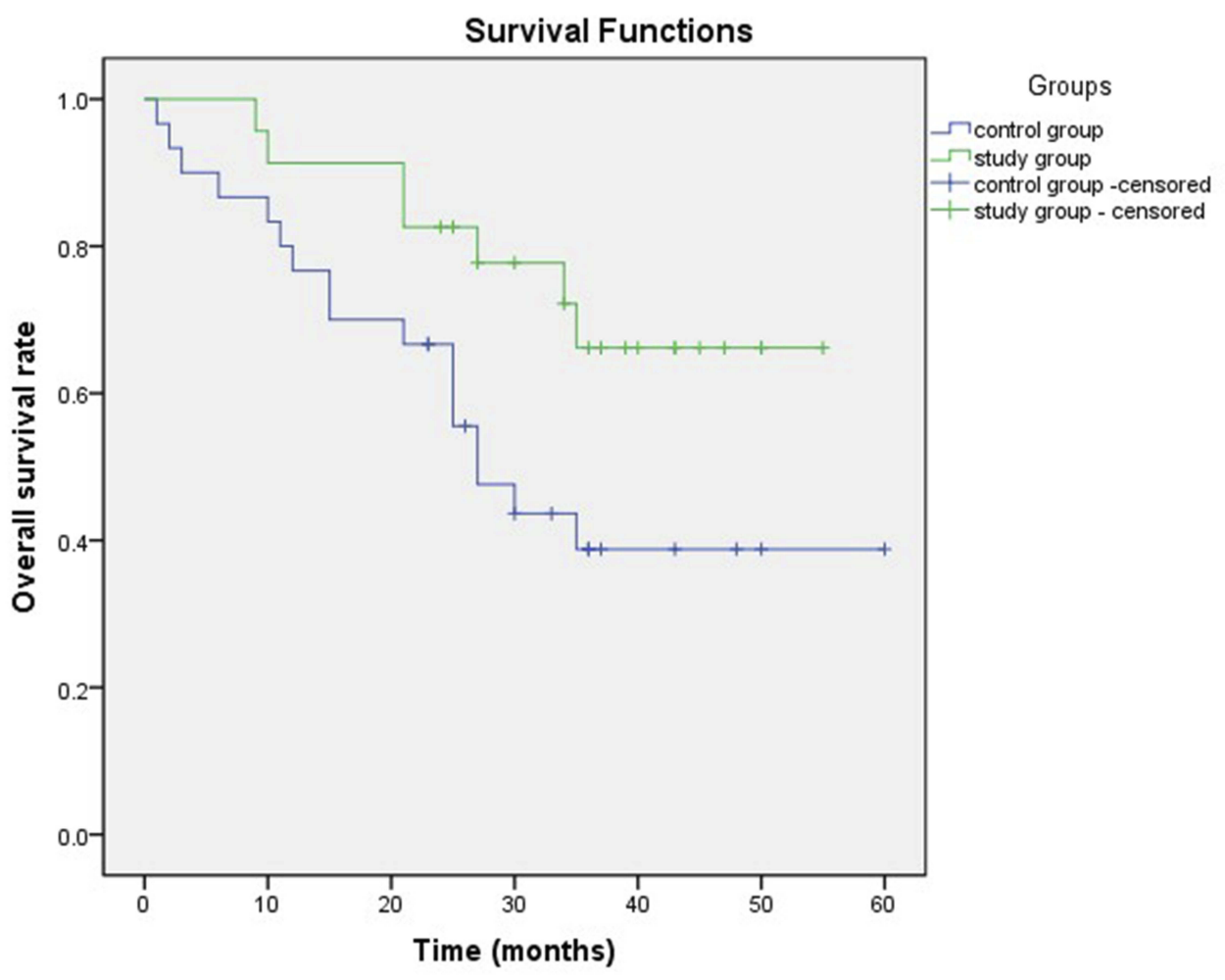

Figure 2 Kaplan-Meier survival curve analysis of progression-free survival in the study and control groups.

Table 3 Comparisons of acute grade 3 and 4 events in the study and control groups [n (\%)]

\begin{tabular}{|l|l|l|l|l|}
\hline \multirow{2}{*}{ Group } & \multicolumn{2}{|l|}{ Hematological system } & \multirow{2}{*}{ Impaired liver or kidney function } \\
\cline { 2 - 4 } & Leukocyte reduction & Thrombocytopenia & Hemoglobin reduction & \\
\hline Study group $(n=23)$ & $9(39.1 \%)$ & $1(4.3 \%)$ & $4(17.4 \%)$ & $2(8.7 \%)$ \\
Control group $(n=30)$ & $21(70 \%)$ & $8(26.7 \%)$ & $14(46.7 \%)$ & $2(6.7 \%)$ \\
$\chi^{2}$ & 5.051 & 4.600 & 4.975 & 0.077 \\
$P$ & 0.025 & 0.076 & 0.026 & 1.000 \\
\hline
\end{tabular}

Table 4 Cox regression model of overall survival

\begin{tabular}{|l|l|l|l|l|l|l|}
\hline Variable & Control & HR & SE & Wald & $\mathbf{P}$ & $\mathbf{9 5 \%}$ CI \\
\hline $\begin{array}{l}\text { Pathological type } \\
\quad \text { Adenocarcinoma }\end{array}$ & Squamous cell carcinoma & & & 1.413 & 0.493 & \\
$\quad$ Adenosquamous carcinoma & & 0.243 & 0.488 & 0.002 & 0.967 & $0.023,2.531$ \\
\hline $\begin{array}{l}\text { Staging } \\
\text { IIla }\end{array}$ & Ilb & 1.020 & 1.267 & 1.286 & 0.257 & $0.392,2.653$ \\
IIlb & & & & 5.963 & $0.05 I$ & \\
\hline Group & & 0.349 & 0.468 & 5.326 & 0.021 & $0.061,2.003$ \\
& Control group & 0.339 & 0.933 & 0.001 & 0.975 & $0.135,0.850$ \\
\hline
\end{tabular}

years. ${ }^{20}$ Other studies have provided evidence of the association between the activated PI3K/AKT/mTOR pathway and cervical cancer, as well as the efficacy of mTOR inhibitor in treating advanced cervical cancer. ${ }^{21,22}$ Only one phase I study treated LACC using mTOR inhibitor, everolimus, cisplatin, and pelvic radiotherapy; however, the results focused 
Table 5 Cox regression model of progression-free survival

\begin{tabular}{|c|c|c|c|c|c|c|}
\hline Variable & Control & HR & SE & Wald & $\mathbf{P}$ & $95 \% \mathrm{Cl}$ \\
\hline Pathological type & Squamous cell carcinoma & & & 1.468 & 0.480 & \\
\hline Adenocarcinoma & & 0.242 & 1.185 & 1.432 & 0.231 & $0.024,2.471$ \\
\hline Adenosquamous carcinoma & & 0.876 & 0.491 & 0.073 & 0.787 & $0.334,2.293$ \\
\hline Staging & Illb & & & 4.599 & 0.100 & \\
\hline IIla & & 0.360 & 0.879 & 1.352 & 0.245 & $0.064,2.015$ \\
\hline Illb & & 0.404 & 0.461 & 3.872 & 0.049 & $0.163,0.996$ \\
\hline Group & Control group & 0.380 & 0.479 & 4.090 & 0.043 & $0.148,0.971$ \\
\hline
\end{tabular}

only on safety, toxicity, and maximum tolerated dose. No further efficacy results have been published. ${ }^{23}$

Apart from the therapies mentioned above, immunotherapy has recently shown progress in the treatment of cervical cancer. The Keynote-028 and Keynote-158 studies investigated pembrolizumab for the treatment of programmed death-ligand 1 (PD-L1)-positive advanced cervical cancer and reported good anti-tumor activity, with an objective response rate of $17 \%$, and safety, even though its activity was limited to PD-L1-positive patients. ${ }^{24,25}$ At present, clinical research on immunotherapy in cervical cancer focuses primarily on recurrent or metastatic cervical cancer. Immunotherapy as a first-line treatment for LACC has not yet been reported. This situation leaves plenty of room to explore more effective approaches.

Nimotuzumab is a humanized monoclonal antibody that competitively binds to EGFR, subsequently blocking downstream signal transduction pathways and cytological effects, which curbs tumor cell proliferation and promotes tumor cell apoptosis. ${ }^{9}$ The principal mechanisms of the increase in radio-sensitivity of tumor cells caused by nimotuzumab include the following: 1) selective recognition and binding to EGFR extracellular domain III, blocking signal transduction pathways led by the EGFR-ligand complex and inhibiting tumor cell cycle and neovascularization, and increasing apoptosis and radio-sensitivity; 2) an anti-tumor efficacy exerted through antibody- and complement-mediated cytotoxicity; 3) downregulation of EGFR to achieve anti-angiogenesis to enhance tumor radiosensitivity. The safety and efficacy of nimotuzumab combined with CCRT have been proven in the treatment of solid tumors such as glioma and head and neck tumors. Another study confirmed that cisplatin-resistant nude mice cervical cancer treated with nimotuzumab combined with CCRT had smaller tumors and longer survival times compared to those in mice treated with either nimotuzumab or
CCRT alone. Mice treated with inotuzumab combined with CCRT also had the most obvious tumor pyknosis and vacuolization phenomena and the lowest expression levels of Akt, p-Akt, MEK, and p-MEK, suggesting that the mechanism might be the result of the blocking of the PI3K/Akt and ERK/MAPK signaling pathways by nimotuzumab. This may be because the blocking can induce cells to reduce cisplatin efflux, enhance cisplatin levels, inhibit DNA replication and transcription, and inhibit the cell division cycle in the G2 and M phases. ${ }^{26}$ Cells normally have the highest radio-sensitivity during these two phases. As mentioned above, in patients with PI3K mutations, the EGFR inhibitor cetuximab had a negative efficacy trend; thus, blocking of the PI3K/Akt pathway by nimotuzumab may have improved efficacy in these patients. One study used nimotuzumab combined with CCRT as a new adjuvant therapy for LACC. After completion, radical hysterectomy and pelvic lymphadenectomy were performed. A total of 28 patients were enrolled, $28.5 \%$ of which achieved complete remission and $71.5 \%$ who achieved partial remission, after completion of neoadjuvant therapy. A total of 24 patients received surgery after neoadjuvant therapy. ${ }^{27}$ However, to date, there has been no published research on nimotuzumab combined with CCRT as a first-line treatment for LACC.

In our investigation, the patients in the study group treated with nimotuzumab combined with CCRT had a significantly higher $\mathrm{CR}$ rate $(78.3 \%)$ than that in the control group $(50 \%)$. There were no statistically significant differences in overall remission and OS rates between the two groups. The PFS in the study group was higher than that in the control group. In addition, comparison of the relative risk using a Cox regression model showed that both FIGO stage and treatment with nimotuzumab affected PFS $(P<0.05)$. Finally, the incidence of nimotuzumabrelated adverse events was low. The main adverse events 
were mild fever, shivering, gastrointestinal reaction, reduced blood pressure, weakness, headache, anemia, etc. The tolerable adverse events experienced in both groups included leukocyte reduction, thrombocytopenia, and impaired liver and kidney functions. Leukocyte reduction differed significantly between the treatment and control groups $(P<0.05)$.

\section{Conclusion}

The combination of nimotuzumab with CCRT in the treatment of LACC resulted in higher CR rates and longer PFS and did not increase the incidence of adverse events compared with treatment with CCRT alone. Due to the high costs, the number of patients enrolled in this study was small, which could have biased the results. A prospective study with larger sample size is needed. Our plan is to continue to supplement the clinical data and provide a reference for subsequent prospective studies to improve treatment options for patients with advanced cervical cancer with the goal of better therapeutic outcomes.

\section{Disclosure}

The authors report no conflicts of interest in this work.

\section{References}

1. Bray F, Ferlay J, Soerjomataram I, Siegel RL, Torre LA, Jemal A. Global cancer statistics 2018: GLOBOCAN estimates of incidence and mortality worldwide for 36 cancers in 185 countries. CA Cancer $J$ Clin. 2018;68:394-424. doi:10.3322/caac.21492

2. William A, Peters III, Liu PY, et al. Concurrent chemotherapy and pelvic radiation therapy compared with pelvic radiation therapy alone as adjuvant therapy after radical surgery in high-risk early-stage cancer of the cervix. J Clin Oncol. 2000;18:1606-1613. doi:10.1200/ JCO.2000.18.10.2059

3. Henry M, Bundy BN, Stenman FB, et al. A comparison of weekly cisplatin during radiation therapy versus irradiation alone each followed by adjuvant hysterectomy in bulky stage IB cervical carcinoma: a randomized trial of the Gynecologic Oncology Group. $N$ Engl J Med. 1999;340:1154-1161. doi:10.1056/NEJM199905133401909

4. Whitney CW, Sause W, Bundy BN, et al. Randomized comparison of fluorouracil plus cisplatin versus hydroxyurea as an adjunct to radiation therapy in stages IIB-IVA carcinoma of the cervix with negative para-aortic lymph nodes: a Gynecologic Oncology Group and Southwest Oncology Group study. J Clin Oncol. 1999;17:1339-1348. doi:10.1200/JCO.1999.17.5.1339

5. Eifel PJ, Winter K, Morris M, et al. Pelvic irradiation with concurrent chemotherapy versus pelvic and para-aortic irradiation for high-risk cervical cancer: an update of Radiation Therapy Oncology Group Trial (RTOG) 90-01. J Clin Oncol. 2004;22:872-880. doi:10.1200/ JCO.2004.08.040

6. Bellone S, Frera G, Landolfi G, et al. Overexpression of epidermal growth factor type-1 receptor (EGF-Rl) in cervical cancer: implications for Cetuximab-mediated therapy in recurrent/metastatic disease. Gynecol Oncol. 2007;106:513-520. doi:10.1016/j.ygyno.2007.04.028
7. Frobe A, Jones G, Bokulic T, et al. High-dose-rate brachytherapy and concurrent chemo-radiotherapy followed by surgery for stage Ib-IIb cervical institution experience. Anticancer Res. 2014;34:3861-3866.

8. Cetina L, Crombet T, Jiménez-Lima R, et al. A pilot study of nimotuzumab plus single agent chemotherapy as second- or thirdline treatment or more in patients with recurrent, persistent or metastatic cervical cancer. Cancer Biol Ther. 2015;16:684-689. doi:10.4161/15384047.2014.987548

9. Gao CZ, Wu XZ, Yan Y, et al. Sensitization of radiation or gemcitabine-based chemoradiation therapeutic effect by nimotuzumab in pancreatic cancer cells. Technol Cancer Res Treat. 2016;15:446452. doi: $10.1177 / 1533034615585209$

10. Basavaraj C, Sierra P, Shivu J, Melarkode R, Monte E, Nair P. Nimotuzumab with chemo-radiation confers a survival advantage in treatment-naive head and neck tumors over expressing EGFR. Cancer Biol Ther. 2010;10:673-681. doi:10.4161/cbt.10.8.12914

11. Boland W, Bebb G. The emerging role of nimotuzumab in the treatment of non-small cell lung cancer. Biologics. 2010;4:289-298.

12. Strumberg D, Schultheis B, Scheulen ME, et al. The emerging role of nimotuzumab in the treatment of non-small cell lung cancer. Invest New Drugs. 2012;30:1138-1143. doi:10.1007/s10637-010-9517-0

13. Rodriguez MO, Rivero TC, Bahi RDC, et al. Nimotuzumab plus radiotherapy for unresectable squamous-cell carcinoma of the head and neck. Cancer Biol Ther. 2010;9:343-349. doi:10.4161/cbt.9.5. 10981

14. Liu ZG, Zhao Y, Tang J, et al. Nimotuzumab combined with concurrent chemoradiotherapy in locally advanced nasopharyngeal carcinoma: a retrospective analysis. Oncotarget. 2016;7:24429-24435.

15. Zheng X, Du X. Prognostic significance of EGFR in cervical cancer: a meta-analysis. Ch J Cancer Prev Treat. 2015;22:1321-1327.

16. Tinker AV, Ellard S, Welch S, et al. Phase II study of temsirolimus (CCI-779) in women with recurrent, unresectable, locally advanced or metastatic carcinoma of the cervix. A trial of the NCIC Clinical Trials Group (NCIC CTG IND 199). Gynecol Oncol. 2013;130:269274. doi:10.1016/j.ygyno.2013.05.008

17. Rose PG, Blessing JA, Lele S, Lele S, Abulafi O. Evaluation of pegylated liposomal doxorubicin (Doxil) as second-line chemotherapy of squamous cell carcinoma of the cervix: a phase II study of the Gynecologic Oncology Group. Gynecol Oncol. 2006;102:210-213. doi:10.1016/j.ygyno.2005.11.048

18. Candelaria M, Arias-Bonfill D, Chavez-Blanco A, et al. Lack in efficacy for imatinib mesylate as second-line treatment of recurrent or metastatic cervical cancer expressing platelet-derived growth factor receptor alpha. Int $J$ Gynecol Cancer. 2009;19:1632-1637. doi:10.1111/IGC.0b013e3181a80bb5

19. Nogueira-Rodrigues A, Moralez G, Grazziotin R, et al. Phase 2 trial of erlotinib combined with cisplatin and radiotherapy in patients with locally advanced cervical cancer. Cancer. 2014;120:1187-1193. doi:10.1002/cner.28471

20. De la Rochefordiere A, Kamal M, Floquet A, et al. PIK3CA pathway mutations predictive of poor response following standard radiochemotherapy \pm cetuximab in cervical cancer patients. Clin Cancer Res. 2015;21:2530-2537. doi:10.1158/1078-0432.CCR-14-2368

21. Hou MM, Liu X, Wheler J, et al. Targeted PI3K/AKT/mTOR therapy for metastatic carcinomas of the cervix: a phase I clinical experience. Oncotarget. 2014;5:11168. doi:10.18632/oncotarget.2584

22. Assad DX, Elias ST, Melo AC, Ferreira CG, De Luca Canto G, Guerra EN. Potential impact of mTOR inhibitors on cervical squamous cell carcinoma: a systematic review. Oncol Lett. 2016;12:41074116. doi:10.3892/ol.2016.5157

23. Melo AC, Grazziotin-Reisner R, Erlich F, et al. A phase I study of mTOR inhibitor everolimus in association with cisplatin and radiotherapy for the treatment of locally advanced cervix cancer: PHOENIX. I Cancer Chemother Pharmacol. 2016;78:101-109. doi:10.1007/s00280-016-3064-0 
24. Frenel JS, Le Tourneau C, O'Neil B, et al. Safety and efficacy of pembrolizumab in advanced, programmed death ligand 1-positive cervical cancer: results from the Phase Ib KEYNOTE-028 Trial. $J$ Clin Oncol. 2017;35:4035-4041. doi:10.1200/JCO.2017.74.5471

25. Schellens JHM, Marabelle A, Zeigenfuss S, Ding J, Pruitt SK, Chung HC. Pembrolizumab for previously treated advanced cervical squamous cell cancer: preliminary results from the phase 2 KEYNOTE-158 study. J Clin Oncol. 2017;35:5514. doi:10.1200/JCO.2017.35.15_suppl.5514
26. Tian SZ, Wang YX, Tian MY, Cheng. Experimental study on the treatment of cervical cancer with drug resistance by nimotuzumab combined with chemotherapy and radiotherapy. Chin J Cancer Prev Treat. 2017;24:1284-1289.

27. $\mathrm{Lu} \mathrm{H}, \mathrm{Wu} \mathrm{Y}$, Liu X, et al. A prospective study on neoadjuvant chemoradiotherapy plus anti-EGFR monoclonal antibody followed by surgery for locally advanced cervical cancer. Onco Targets and Ther. 2018;11:3785. doi:10.2147/OTT.S164071

\section{Publish your work in this journal}

Cancer Management and Research is an international, peer-reviewed open access journal focusing on cancer research and the optimal use of preventative and integrated treatment interventions to achieve improved outcomes, enhanced survival and quality of life for the cancer patient.

Submit your manuscript here: https://www.dovepress.com/cancer-management-and-research-journa
The manuscript management system is completely online and includes a very quick and fair peer-review system, which is all easy to use. Visit http://www.dovepress.com/testimonials.php to read real quotes from published authors. 